\title{
Trauma in pregnancy
}

\author{
Udo Rudloff
}

Received: 4 December 2006 / Accepted: 11 December 2006 / Published online: 12 January 2007

(C) Springer-Verlag 2006

\section{Introduction}

The magnitude of the problem

Trauma of pregnant women with its potential impact onto the health of both the mother and the fetus has evolved over the last decades into a major adverse risk factor to successful pregnancy outcome. Trauma now represents the leading cause of non-obstetric causes of death in pregnancy, accounting for $6-7 \%$ of all maternal deaths $[1,2]$. For certain inner city socioeconomic and ethnic groups, however, rates as high as $20-46 \%$ have been reported [3].

Overall, approximately 1 out of 14 pregnant women will experience some sort of significant trauma during their pregnancy. Three to four out of 1,000 pregnant patients will require ICU care for their injuries. Fetal mortality mirrors the severity of maternal injuries and has been reported as high as $61 \%$ in women with major trauma and $80 \%$ if maternal shock is present $[4,5]$. The main cause of fetal death is maternal death secondary due to blunt abdominal trauma, the second most common placental abruption followed by actual uterine rupture $[4,6]$.

Motor vehicle accidents (MVAs) account for more than $50 \%$ of blunt trauma in pregnancy, followed by falls and assaults (Table 1) [2]. Motor vehicle accidents contribute also for the majority of maternal and fetal deaths $[1,2]$. Although penetrating maternal trauma

U. Rudloff $(\square)$

Department of Surgery,

New York University Medical Center, BVH, 15N1, 462 First Avenue, New York, NY 10016, USA e-mail: rudlou01@med.nyu.edu accounts only for $5 \%$ of overall maternal death, it has been reported to be associated with fetal demise in up to $60 \%$ of cases [6]. Minor maternal injuries should not decrease the physician's awareness of the specific needs of the pregnant trauma victim since they are associated with a $5 \%$ incidence of placental abruption or fetomaternal hemorrhage [7].

The obstetrician and trauma surgeon should be aware that there exists a specific risk distribution of trauma during the trimesters of pregnancy. While falls happen frequently between 20 and 30 weeks, trauma due to domestic abuse and interpersonal violence becomes more prevalent as the pregnancy progresses [1]. MVAs were reported to occur with equal frequency throughout pregnancy.

Domestic violence in pregnancy has found recently attention in several contributions to the scientific and lay literature [8-10]. Emergency department records suggest that $22-35 \%$ of women presenting with any complaint have in fact injuries related to physical abuse [11]. Some series report concerning incidence rates of domestic physical abuse of $31.5 \%$ during pregnancy [8]. These numbers, together with an increased public awareness, have let to the introduction of screening measures for domestic violence (questionnaires, midwifery training) into the antenatal care of US and UK centers $[12,13]$.

The implementation and adherence to standard protocols and expert guidelines striving for standardization of care has been shown in obstetric and surgical fields to improve outcome and reduce mortality and morbidity. For pregnant trauma victims, evidencebased algorithms have only recently been developed by few panels and organization, and have not yet undergone a rigorous audit and review process. 
Table 1 Incidence of various types of blunt trauma in pregnancy

\begin{tabular}{ll}
\hline Type of blunt trauma & Total number $(\%)$ \\
\hline $\begin{array}{l}\text { Motor vehicle accidents/ } \\
\text { pedestrian struck }\end{array}$ & $1,098(59.6)$ \\
Falls & $411(22.3)$ \\
Direct assaults & $308(16.7)$ \\
Other & $24(0.1)$ \\
\hline
\end{tabular}

This article reviews the recently released guidelines of the Eastern Association for the Surgery of Trauma (EAST) on trauma in pregnancy [14]. It focuses in the context of the established ATLS protocols for the care of pregnant trauma victims on the specific pathophysiologic characteristics of injured pregnant patients. In reference to recently introduced new concepts in trauma surgery it includes brief discussions on the concept of damage control surgery, penetrating trauma, injury scoring, and pelvic fractures in injured pregnant women.

\section{Pre-hospital care}

Misconceptions of healthcare workers, unfamiliarity with the specific changes of pregnancy, and an increased fear and anxiety level of the patient or family have hampered prompt and effective resuscitation of pregnant trauma patients in the past. The possibility of pregnancy should be entertained in all women of childbearing age as early as possible, and any information about gestational age should be obtained and related to the receiving institution. Pregnancy is considered a triage criterion for transport to a trauma center by the American College of Surgeons Committee on Trauma. Despite this recommendation, literature on the appropriate level of care in injured pregnant patients is very limited. Goodwin and Breen [15] proved in a landmark contribution in 1990 that, in addition to the accepted ATLS guidelines for transferal of patients to level I trauma centers (like Glasgow Coma Score less than 14, respiratory rate less than 10 or greater than 29 , systolic blood pressure less than 90, Revised Trauma Score (RTS) less than 11, anatomy or mechanism of injury) that in pregnant trauma victims a pulse rate faster than 110 , chest pain, loss of consciousness, and third trimester gestation are independently correlated with need for trauma center. These criteria are particularly useful in mass casualty triage of patients in adjunction to prehospital trauma scoring systems in order to identify those patients who would benefit most from rapid transfer to trauma centers.

In general, guidelines for adult pre-hospital trauma care also apply to pregnant trauma victims. Upon initial assessment EMS should follow standard protocols like extrication with spinal immobilization and resuscitation as outlined in the ATLS guidelines. The decision to intubate the patient in the field is largely unaffected by pregnancy. Unique to the gravid patient in airway management are, however, as follows:

1. Pregnant patients are at increased risk of aspiration due to delayed gastric emptying and decreased lower esophageal sphincter tone in combination with intra-abdominal hypertension.

2. Although the safety of rapid-sequence intubation is well established in pregnancy, because of lower serum pseudocholinesterase levels in pregnancy, using a lower dose of succinylcholin during induction is recommended [16].

3. Both depolarizing and non-depolarizing muscle relaxants cross placenta. Effects of these drugs onto CTG pattern and fetal activity might lead to a falsely non-reassuring tracing and non-indicated intervention. In the event of delivery, the neonatologist might be faced with a flaccid, apneic infant. Hence, it is pertinent to relate any pre-hospital use of medication by EMS to the receiving institution and trauma team.

In general, the potential catastrophic consequences of the patient loosing her airway in the field or during transport usually justify acceptance of the minor risks associated with using paralytic and induction agents. Early establishment of a definite airway is usually the safest option.

Avoidance of the supine hypotension syndrome (uterocaval compression) should be paramount part of all initial resuscitative measures in pregnant trauma patients. Placing the patient on a backboard with a $15^{\circ}$ angle to the left is a pregnancy-specific intervention that should be employed in all patients beyond 20 weeks of gestation. Abundant clinical data have proven that the significantly decreased cardiac output of up to $60 \%$ due to uterocaval compression leads to prolonged resuscitation with increased acidosis and vasopressor requirements [17, 18]. Below 24 weeks manual lateral displacement might be sufficient in gestations of more than 24 weeks a $30^{\circ}$ lateral tilt is recommended. Although this reduces the efficacy of cardiopulmonary resuscitation (CPR) compared to the supine position, in pregnant patient the slightly reduced efficacy of chest compressions is outweighed by improved cardiac preload and overall cardiac output [17]. Therefore, current EAST guidelines now recommend a left lateral tilt of at least $15^{\circ}$ during the initial phase of resuscitation.

Placement of a hard backboard in the supine position might not be tolerable for third trimester gravida. 
The increased work of breathing due to increased diaphragmatic splinting might lead to respiratory failure. In this circumstance, transport in a $30^{\circ}$ reversed Trendelenberg position seems acceptable [19].

As far as IV access routes in pregnant trauma patients are concerned, femoral access procedures should be avoided. Because of the risk of uterocaval compression, distribution of medication or fluids might be significantly altered when using the femoral route in pregnant patients.

\section{Primary survey}

After arrival in the ED, during the primary survey, immediate life-threatening conditions are identified and corrected. Oxygenation and oxygen delivery with establishment of adequate tissue perfusion is the primary goal of the primary survey

A prepared and pre-alerted multispeciality team should receive the patient in the ED. As much information as possible about the pre-hospital resuscitative phase should have been provided. A multidisciplinary approach with the ED physician, trauma surgeon, and obstetrician being present is frequently required. Prehospital care and further resuscitation including airway management is most often directed by the ED physician. In close consultation with the trauma surgeon and obstetrician further resuscitation, diagnostic studies, or operative intervention including delivery is simultaneously planned and instituted in order to maximize both maternal and fetal outcome. Figure 1 depicts the role of the different specialties in the multidisciplinary approach to trauma in pregnancy emphasizing the simultaneity of decision making processes between the different specialties.

Gravid patients have a significantly decreased $\mathrm{O}_{2}$ reserve. Hypoxemia secondary due to respiratory or cardiovascular failure occurs significantly earlier than in non-pregnant patients. Several different factors and adaptations of pregnancy account for this decreased ability of gravid patients to withstand episodes of decreased oxygen delivery:

1. Oxygen demand and consumption in pregnant women is increased at rest by $20 \%$ due to the
Fig. 1 Multidisciplinary decision-making in the case of the "two-in-one" victim of trauma

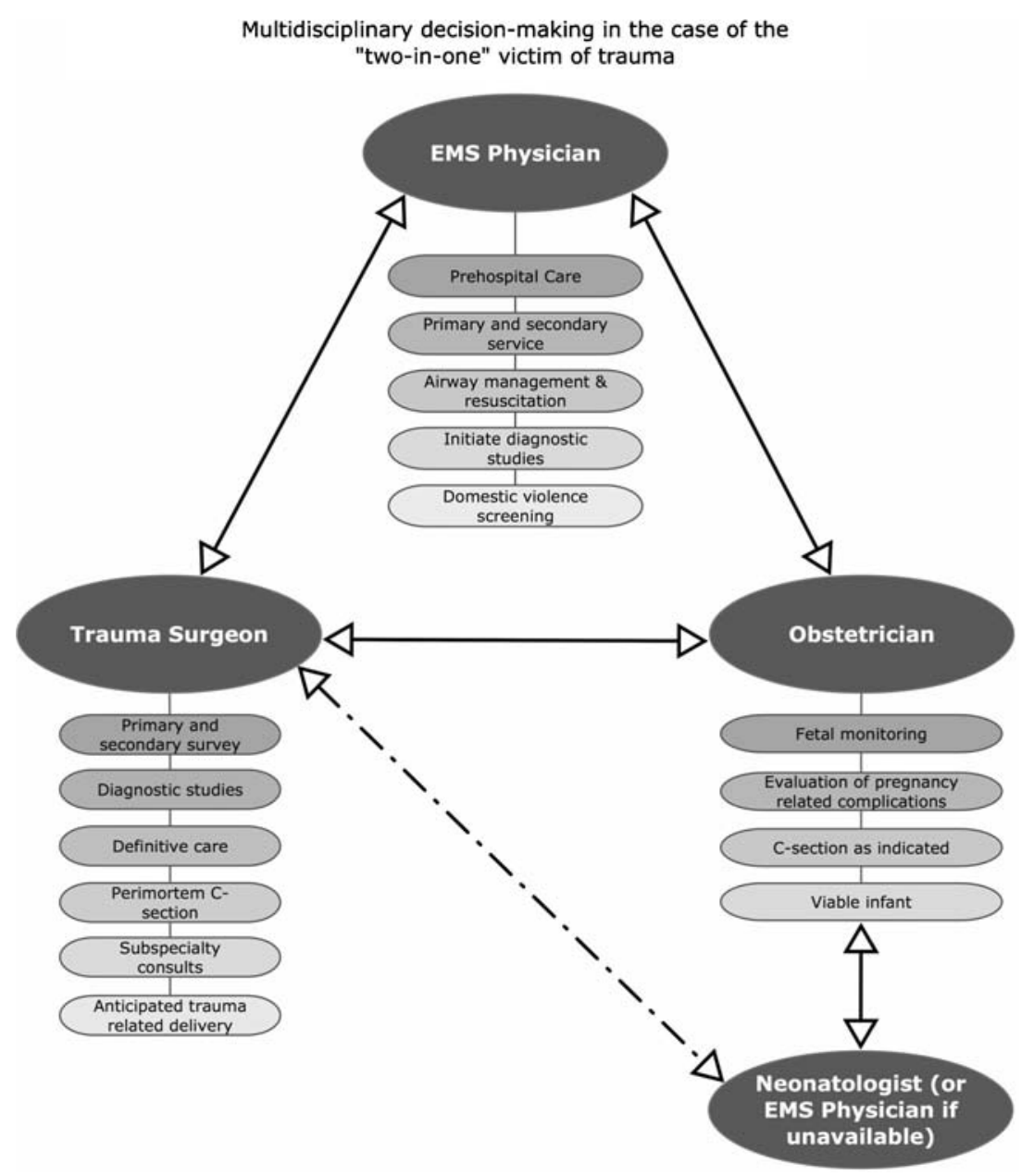


metabolic demands of growing uterus, fetus, placenta, and breasts. Increased 2,3-DPG levels facilitate oxygen de-loading and placental transfer, but leave the mother with a decreased oxygen reserve. Overall, mean arterial oxygen tension is decreased by $29 \%$ in third trimester of pregnancy. Maternal compensatory mechanisms for the reduced oxygen binding include an increase in minute ventilation and an increase of maternal cardiac output but leave the reduced maternal oxygen reserve unchanged [19].

2. Maternal compensation of periods of hypoxemia is also hampered by a decreased respiratory reserve during pregnancy. Owing to elevation of the maternal diaphragm starting in the second trimester of pregnancy, maternal expiratory reserve volume starts to decrease leading to a $18 \%$ reduction of FRC in the third trimester of pregnancy. The limited expiratory reserve volume renders pregnant patients more intolerant to episodes of apnea [20]. In view of the extreme sensitivity of the fetus to hypoxia with fetal shunting ('diving reflex') occurring at maternal oxygen levels $\left(\mathrm{p}_{\mathrm{a}} \mathrm{O}_{2}\right)$ of 60 $\mathrm{mmHg}$, the limited maternal compensatory ability to withstand episodes of hypoxia makes maintenance of adequate oxygen levels in the mother mandatory. Despite the absence of class I evidence, all pregnant patients should be put on supplemental oxygen to increase the error of margin.

Several anatomic and physiologic changes of pregnancy may impact significantly on the primary survey in gravid trauma patients:

\section{Airway}

On arrival of the pregnant trauma patient in the emergency department, the primary survey should start with re-assessment of the patient's airway. Any altered level of consciousness, Glasgow Coma Score levels of less than 8 , findings of respiratory distress, or non-purposeful motor responses should lead to establishment of a definite airway in cervical spine protection on arrival.

Although decreased lower esophageal sphincter and gastric tone, cephalad displacement of intra-abdominal organs, and increased gastric acid production put pregnant patients at increased risk of developing aspiration syndrome, the routine placement of either oro- or nasogastric tubes is not recommended.

\section{Breathing and ventilation}

Immediately following the assessment and establishment of airway, functions of lungs, chest wall, and diaphragm are assessed to rule out acutely life-threatening conditions like pneumothorax, tension pneumothorax, cardiac tamponade, or flail chest.

Injuries that may acutely impair ventilation in pregnant women are more difficult to diagnose. A certain degree of tachypnea and respiratory difficulty ("air hunger") is not abnormal in the third trimester and may confuse serious underlying lung injury. Owing to limited thorax excursions, conditions like flail chest or pneumothorax are more difficult to detect in pregnancy, and because of diminished respiratory reserves with reduced expiratory volumes and functional residual capacity may lead in pregnant trauma patients sooner to decompensation. Any tube thoracostomy for pneumo- or hemothorax needs to be placed one to two intercostals spaces higher in pregnant patients to avoid diaphragmatic injury.

\section{Circulation with hemorrhage control}

Assessment of circulation and interpretation of vital signs should also be done in the context of pregnancy-related changes. Gravid patients have from the early second trimester an abundant physiologic reserve to compensate for fluid loss and hemorrhage. They often demonstrate few signs of hypovolemia even after severe volume depletion. When deterioration does occur, it is often precipitous and catastrophic. Therefore, hypotension occurring in pregnant trauma victims should be regarded an ominous sign requiring urgent intervention [21].

The physiologic anemia of pregnancy is most pronounced at the end of the second trimester. Physiologically another mechanism of self-preservation against peri-partum blood loss, it can significantly mislead the treating physician. Maternal blood volume increases by $50 \%$, but maternal erythropoiesis does not keep pace (10-15\% increase in red blood cell mass only). Owing to the expansion of blood volume hemodynamic instability occurs only when blood loss approaches 1,500 to $2,000 \mathrm{cc}$ [21]. Recruitment of intravascular volume to compensate for losses will instantly be at the expense of the low resistance uteroplacental unit. The reason for the extraordinary sensitivity of the uteroplacental unit to maternal hypovolemia lies in the high expression of catecholamine receptors in the large venous sinuses in the placental bed and uterine wall. At the end of pregnancy, the density of alpha receptors of the intramyometrial vessels has increased significantly.

Therefore, fetal distress is often the first sign of maternal hemodynamic compromise, and generally occurs well before change in maternal vital signs. This maternal response to stress as a mechanism of selfpreservation at the expense of the fetus may be the 
most accurate indicator of maternal health. Because of its sensitivity, and in the absence of other reliable indicators, fetal well being is considered an endpoint of resuscitation in pregnant patients.

Early crystalloid fluid replacement and aggressive transfusion of blood products is essential to restore volume and increase oxygen delivery. Despite the low oncotic pressure in pregnant women as a consequence of the physiologic anemia, there is no evidence supporting a preferred role of colloids or albumin in the early resuscitation phase. New promising strategies like resuscitation with hypertonic saline (e.g., head injured patients), or synthetic hemoglobins have not been tested in pregnancy at this point.

If blood is needed before a full cross-match is complete, it should be Rhesus (Rh) factor negative. Other blood factors, including fresh frozen plasma and cryoprecipitate should be readily available in view of the increased risk of pregnant trauma patients developing DIC. Recently, several reports have also shown a role of Factor VII in the management of hemorrhagic shock in pregnancy $[22,23]$.

Other endpoints of resuscitation in pregnant trauma patients other than fetal health deserve additional consideration:

Maternal acidosis has been suggested to accurately reflect maternal fluid status, and maternal acidosis is a predictor of fetal demise [24-26]. However, interpretation of arterial blood gas (ABG) analysis requires knowledge of the specifics of acid-base-status in pregnant women. Early in the second trimester of pregnancy increased tidal volumes and respiratory rate lead to a mild respiratory alkalosis with $\mathrm{pCO}_{2}$ levels generally below $32 \mathrm{mmHg}$. Compensatory excretion of bicarbonate by the kidney decreases circulating bicarbonate levels. In the event of increased production of lactate, like in hemorrhagic shock, the oxygen deficit manifest through arterial serum acidosis will lead to an earlier change in $\mathrm{pH}$ compared to the non-pregnant patient population. Calculation of the base excess in these circumstances is accurate and mandatory when evaluating patients with suspected hemorrhage. Serial serum lactate measurements are considered by many authorities the current gold standard for assessment of successful resuscitation, but might be confounded in pregnancy. In pregnant patients, the increased glomerular filtration rate can lead to low levels of serum lactate delaying the development of lactic acidosis and decreasing the sensitivity of this parameter as an endpoint of resuscitation. On the other hand, renal and liver disease specific to pregnancy, like pre-eclampsia or HELLP syndrome, might either interfere with renal excretion or hepatic metabolism of lactate leading to falsely elevated levels.
Elevated lactate levels taken immediately after commencement of resuscitation also do not accurately reflect an oxygen deficit in these patients. A transient rise in serum lactate washed out from the capillary bed is often seen, and does not reflect unresponsiveness of the patient to the resuscitative efforts.

New developments like sublingual capnography as part of targeted resuscitation strategies after injury have not been studied in pregnant patient population [26]. Sublingual capnography accurately assesses blood flow in the gastrointestinal tract by measuring $\mathrm{CO}_{2}$ content spectroscopically. Increased levels of $\mathrm{CO}_{2}$ in the sublinguinal veins, which are part of the splanchnic circulation, indicate decreased perfusion of the intestines. Since shunting of blood away from the splanchnic circulation occurs very early in any hypovolumic states, this method of assessing the perfusion of the gastrointestinal organ system is thought to be very sensitive to detect fluid deficits or ongoing fluid losses. If the relative abdominal hypertension of pregnancy requires re-definition of reference ranges has not been investigated at this point.

\section{Disability (neurologic examination)}

Head injury is the most frequent cause of maternal death in injured pregnant women [27, 28]. Like in the non-pregnant trauma patient, in the absence of hypoxia and hypovolemia any changes in the mental status or level of consciousness should be thoroughly investigated for head trauma and central nervous system injury. Conditions of pregnancy, like eclampsia, have to be included into the differential diagnosis and may impede diagnostic accuracy. Alcohol or drug ingestion occurs in a significant proportion of pregnant trauma patient [29]. Illicit substance abuse will not only affect the sensorium of the mother but might also flaw the diagnostic accuracy of fetal monitoring.

\section{Exposure/environmental control}

The same principles as far as complete exposure of the trauma patient with examination of the back apply to pregnant women. The trauma team should be cogniscent of the development of significant hypothermia. In case trauma has induced labor, and delivery appears imminent, all clothing should be removed to prevent asphyxiation of the fetus in case of precipitous delivery.

\section{Fetus (peri-mortem cesarean section)}

Peri-mortem cesarean section refers to operative delivery of the fetus which is performed at the time or after 
maternal death. Several authorities recommend delivery by cesarean if there is in the absence of vital signs no response to CPR within $5 \mathrm{~min}$, and any correctable life-threatening injury during primary survey had been ruled out [30-32]. Peri-mortem section should be initially perceived as part of maternal resuscitation, and not prioritization of fetal outcome over maternal outcome. The increased venous return that occurs after the uterus has been emptied optimizes the potential outcome for both the mother and the fetus. In particular, the pooling of blood, detrimental to maternal resuscitation, in the low-resistance, large-capacitance circulation of the uteroplacental unit is abolished. Following the landmark reviews of Katz et al. and Morris et al. EAST guidelines now recommend peri-mortem cesarean section should to be performed in any moribund pregnant woman of more than 24 weeks of gestation [14, 31, 33]. Peri-mortem cesarean section must occur within $20 \mathrm{~min}$ and should start within $4 \mathrm{~min}$ of maternal arrest. It is recommended to perform a large midline incision from the xiphoid process to the symphysis pubis followed by a vertical incision of the uterus from the fundus into the lower segment. The mortality and fetal neurological outcome is closely linked to the length of the adynamic arrest of the mother and the time interval between maternal arrest and delivery $[30,31]$. With timely delivery a fetal survival rate greater than $60 \%$ can be expected with favorable neurological outcome in most of the cases.

\section{Secondary survey}

The main aim in the treatment of the injured pregnant patient is to stabilize the mother first then turn attention to the fetus. This paradictum emerged from the fact that, still up to date, the most common cause of fetal death is maternal death. However, the health assessment of the fetus should be used early as an invaluable tool in the maternal response to resuscitation.

The secondary survey, the head-to-toe evaluation of the trauma patient, is a complete history and physical examination of each region of the body with constant reassessment of all vital signs. It only starts after the primary survey is completed, resuscitative efforts are established, and the patient demonstrates response to resuscitation. In major trauma, it should be carried out by the trauma surgeon in conjunction with the obstetrician. The secondary survey is a continuation of the initial resuscitative efforts, since it aims to identify any underlying not yet recognized injury imminently hazardous to the patients' survival.
A detailed history about the particular circumstances of the incident may provide valuable clues to specific injuries. The mechanism of the injury should be known at the start of the secondary survey, and it is particularly important in evaluating head, thoracic, and abdominal trauma. Examples are direction of impact and vehicular intrusion, deployment of air bags, excitrication, or bent steering wheels.

The following review of organ systems focuses on clinically relevant pregnancy-related changes of pregnant trauma patients:

\section{Thoracic trauma}

Management of thoracic trauma in pregnancy is similar to the non-pregnant patient population. Potential pitfalls due to the anatomical and physiological changes in pregnancy are as follows:

1. Chest tubes for hemopneumothoraces need to be because of elevation of the diaphragm placed one to two intercostals spaces higher.

2. Evaluation of blunt cardiac trauma is hampered by EKG changes due to pregnancy. Left axis shift, flattened T-waves in anterior chest leads, presence of $\mathrm{Q}$ waves in leads III and avF can be normal in the third trimester of pregnancy and be confused with EKG changes due to cardiac contusion. Early use of echocardiography is recommended to screen for cardiac contusion in pregnant trauma patients.

3. Elevation of the diaphragm in pregnant women (up to $4 \mathrm{~cm}$ ) with widening of the cardiac shadow and mediastinum reduces the diagnostic accuracy of plain chest films for aortic injury. Transesophageal echocardiography and helical CT scanning of the chest are the diagnostic tests of choice to rule out thoracic aortic injury or traumatic pericardial tamponade.

4. The risk of concomitant abdominal injuries in transversing penetrating thoracic trauma is increased due elevation of the diaphragm.

5. Owing to reduced inspiratory efforts and decreased lung volume in pregnant patients small pneumothoraces are more likely to be missed on initial plain chest radiographs. Repeat films are often required to rule out pneumothoraces.

6. Because of the decreased oxygen reserve of pregnant women pulmonary contusion in pregnant patients requires more frequently intubation and ventilatory support. It can present with a wide spectrum of clinical signs including confusion or signs of fetal distress. The severity of the lung injury is usually not well correlated with the find- 
ings on chest radiographs which are often initially normal. Suspected pulmonary contusion in pregnant patients requires careful monitoring with a low threshold for early intubation and mechanical ventilation.

7. In the absence of specific guidelines for the performance of ED thoracotomies in blunt and penetrating thoracic trauma, the role of emergency thoracotomy in pregnant trauma patients remains to be defined. A recent recommendation by a report of Lee et al. [34] to perform open cardiac massage without cross-clamping of the thoracic aorta in all pregnant patients without any obvious thoracic injuries who do not respond to CPR has not found wider acceptance. Emergency thoracotomy might have extended indications in pregnancy, and should be performed in conjunction with peri-mortem cesarean section in cases of more than 24 weeks of gestation.

\section{Abdominal trauma}

\section{Blunt abdominal trauma}

Blunt abdominal trauma in pregnant women is the number one cause of maternal and fetal death due to trauma in pregnancy. Fetal death is most commonly due to maternal death followed by placental abruption $[5,6,19]$. Motor vehicle accidents account for $60 \%$ of blunt traumas [1]. Pregnant women are less likely than other motorist to wear seatbelts because of concerns of fetal injury [35, 36]. If they wear seatbelts they are often applied over the wrong site, potentially leading to increased fetal mortality [36]. The ACOG and the RCOG have now produced specific guidelines reassuring pregnant women about the use of seatbelts reviewing the safety and benefit of correctly applied three-point safety restraints $[37,38]$.

One of the most striking and challenging differences between blunt abdominal trauma in pregnant and nonpregnant patients is the reduced diagnostic accuracy and sensitivity of the physical examination in pregnant trauma patients with intra-abdominal injuries compared to the non-pregnant population. The muting of peritoneal irritation in pregnant trauma patients, which significantly confounds the physical examination of pregnant women with blunt abdominal trauma, is due to the following:

1. The chronic stretching of the parietal peritoneum by the growing uterus, in particular in the third trimester, decreases the number of afferent sensory nerve fibers of the peritoneum per square centimeter $\left(\mathrm{cm}^{2}\right)$. This leads to an increased threshold for peritoneal excitation secondary due to intraperitoneal bleeding or other pathological processes, and explains the frequent absence of signs of peritoneal irritation in the third trimester of pregnancy.

2. Pain due to intra-abdominal processes may be referred to atypical locations due to cephaled displacement of intestinal organs secondary to the growing uterus in pregnancy.

3. In the later stages of pregnancy, the cephaled compression of intestinal organs leads to interposition of omentum and small intestines between the parietal peritoneum and any intra-abdominal process. This shielding of peritoneal irritation contributes further to the reduced reliability of physical findings in pregnant trauma patients.

Maternal injuries Splenic injury and retroperitoneal bleeds are the most common injuries due to blunt abdominal trauma of pregnancy [39]. Both injuries occur significantly more frequently than in non-pregnant women.

Overall, the most common cause of intraperitoneal hemorrhage in pregnant trauma patients is splenic injury. The engorged organ with its increased vascularity is believed to rupture more early than in the nonpregnant state [39]. Several reports describe higher grade splenic injuries at laparatomy compared to the non-pregnant patients $[39,40]$. Non-operative management is also the most commonly pursued approach for splenic rupture in pregnant patients. These findings are in contrast to liver injuries where liver seize and blood flow are unaffected by pregnancy. However, several recent reports suggest that certain pregnancy induced conditions, like HELPP syndrome, carry an increased risk of hepatic rupture and bleeding in pregnant trauma patients [41, 42].

In the later stages of pregnancy, retroperitoneal bleeds are a feared and frequent consequence of blunt abdominal trauma [19, 39]. The sudden increase of intra-abdominal pressure leads to rupture of the congested, engorged pelvic venous plexus, fed by the uteroovarian and infundibulopelvic vessels, into the retroperitoneum [43]. Traumatic retroperitoneal bleeds are often associated with consumptive coagulopathy and have a high incidence of fetal death [40]. Massive substitution of blood products is often required and a non-operative approach is initially usually pursued. In order to gain access and exposure of the retroperitoneum, emergency cesarean section and delivery might be required, and is indicated under these circumstances.

Pregnant patients also have an increased risk of bladder injury. The cephaled displacement of the 
bladder by the growing uterus, becoming in intraabdominal organ in the second trimester of pregnancy, increases the risk of bladder rupture in cases of blunt abdominal trauma. Many centers have abandoned diagnostic peritoneal lavage (DPL) for the evaluation of blunt abdominal trauma in favor of the less invasive focused abdominal sonogram of trauma (FAST) examination. Several reports confirm its equal sensitivity in detecting intraperitoneal fluid [44, 45]. However, with its ability to distinguish between blood, succus entericus, or urine in cases of intra-abdominal fluid, some trauma surgeons prefer DPL. A supraumbilical approach should be chosen in pregnant patients. It is also recommended to use an open technique and to avoid inadvertent puncture of the uterus during Seldinger technique.

Injuries of the uterus, fetus, and placenta In the stable pregnant patient thorough assessment of the uteroplacental unit and the fetus is an incremental and important part of the secondary survey. It serves to identify specific, potentially life-threatening injuries to the uterus and the fetus, and provides the trauma surgeon with the unique opportunity to use the status of the fetus as a very sensitive reflector of maternal health.

Serious injuries to the uterus, placenta, or fetus may present with very subtle signs. Any discrepancy between the fundal height estimated by gestational age and the actual height of the uterus on physical examination might be the only hind of uterine rupture. Any subtle signs of uterine tenderness, contractions, or uterine irritability, either on clinical examination or on tocographic monitoring, should raise the suspicion of placental abruption in the context of blunt abdominal trauma. Vaginal bleeding might discern placental abruption, uterine rupture, rupture of membranes, or pelvic fracture with vaginal injury.

Fetal monitoring: The duration of continuous fetal monitoring (CFM) in the evaluation of pregnant trauma patients has recently been re-defined. EAST guidelines now recommend that all pregnant women of more than 20 weeks after any kind of trauma have CFM for at least $6 \mathrm{~h}$ [14]. Monitoring should then be continued depending on the clinical situation. Further evaluation should be carried out if uterine contractions, a non-reassuring fetal heart rate pattern, vaginal bleeding, significant uterine tenderness or irritability, serious maternal injury, or rupture of the amniotic membranes is present. The basis for these recommendations stems from the observation that abruptio placentae can occur up to $72 \mathrm{~h}$ post-injury including minor trauma $[46,47]$. Fetal outcome after $2-6 \mathrm{~h}$ of monitoring is in the absence of symptoms usually excellent.
The combination of assuring fetal heart rate tracing and no clinical symptoms has negative predictive value of near $100 \%$ for adverse outcome [1]. Studies examining the role of adding further elements of the physical profile to the initial fetal evaluation in pregnant trauma patients failed to demonstrate an increase in accuracy in regard to assessing fetal well being [48]. The trauma team needs to be aware of the limitations of ultrasonography in these evaluations. The sensitivity of sonography to identify post-traumatic abruption is only $40-50 \%[7,49]$. Therefore, the absence of visualization of subchorionic hemorrhage or a retroplacental clot on ultrasonography in the presence of clinical symptoms like abdominal pain mandates further CFM with a low threshold to intervene should signs of fetal distress appear.

There has been a great interest in using laboratory markers in aiding the diagnosis of placental abruption. Elevated D-dimer levels and fibrin-split products (FSP) are early markers for accelerated fibrinolysis in beginning dissiminated intravascular coagulopathy (DIC). Although often found to be elevated in cases of placental abruption, most studies failed to demonstrate clinical usefulness as a screening test $[7,50]$.

Fetal-to-maternal hemorrhage: Another screening test suggested for the detection of placental abruption is the Kleihauer-Betke (KB) test measuring the amount of fetal hemoglobin in the maternal circulation. It estimates the amount of fetomaternal hemorrhage by the formula 1 fetal red blood cell per 1,000 maternal cells corresponding to $1 \mathrm{cc}$ of fetomaternal hemorrhage. Although one study showed an increased incidence of placental abruption in those with a positive test, several large series have now let to the consensus that the low specificity and poor negative predictive value significantly limits the clinical usefulness of the Kleihauer-Betke test as a screening test for placental abruption [6, 49, 51].

Nevertheless, positive KB results with signs of fetal distress should alert the obstetrician of massive fetomaternal hemorrhage potentially requiring intrauterine transfusion if delivery is not warranted due to prematurity [51]. In a recent analysis a positive Kleihauer-Betke test was a predictor of pre-term delivery [33]. Its main purpose in the evaluation of the pregnant trauma patient lies in the quantification of any fetomaternal hemorrhage, and to restrict Rhesus immune globulin administration to those who need it including the few patients for whom the initial dose is insufficient [30]. EAST guidelines recommend $\mathrm{KB}$ analysis in all pregnant patients involved in trauma who are more than 12 weeks of gestation [14]. Thirty microgram of Rhesus immune globulin per $30 \mathrm{cc}$ fetomaternal hemorrhage 
should be given, and repeat doses might be necessary within the $72 \mathrm{~h}$ window. As per ACOG guidelines, another option is to administer Rhesus immune globulin to all non-sensitized Rhesus negative women and guide additional dosing according to the $\mathrm{KB}$ test results.

Placental abruption: One of the most feared sequelae of trauma in pregnancy from a fetal perspective is placental abruption. It represents the second most common cause of fetal mortality in trauma patients, and its incidence has been reported to vary between 20 and $50 \%$ or higher in mothers who survive major trauma $[15,26]$. Minor trauma carries a risk of placental abruption estimated as high as $1-5 \%[7,15$, 26]. Understanding the causative mechanism of placental abruption will remind the treating physician about the risk of delayed presentation of this condition: The sudden increase in intra-abdominal pressure in blunt trauma leads to acute compression and deformation of the elastic myometrium. This exerts shearing effects on the rigid, fibrous placental villi what frequently leads to rupture of adjacent, small placental sinuses. A small retroplacental clot will form initially. Owing to the high thromboplastin concentration in the surrounding trophoblastic tissue local DIC is likely to develop leading to expansion of the clot. Ultimately, complete placental separation and fetal demise will occur as the process continues. Depending on the seize of the initial clot, placental abruption might not be infrequently asymptomatic in its early stages. However, with expansion of the retroplacental clot uterine activity will usually emerge. With more than eight registered contractions per hour and in the absence of reassuring fetal monitoring, there is a $25 \%$ chance of finding placental abruption in a pregnant women after blunt abdominal trauma [49]. Only $35 \%$ of clinically significant placental abruptions had vaginal bleeding [15]. Although placental abruption is frequently found in association with fetomaternal hemorrhage, screening for fetal blood cells in the maternal circulation with Kleihauer-Betke tests has a low specificity and is currently for this purpose not recommended [1, 30]. Screening should be performed with CFM which has in the absence of clinical symptoms, and when performed over $6 \mathrm{~h}$, an excellent negative predictive value [27, 49]. In view of the variable presentation of placental abruption, it is paramount to be aware that placental abruption can occur up to $48 \mathrm{~h}$ after the initial trauma. Although some of the described delayed cases might have been missed diagnoses, current guidelines emphasize in the presence of clinical symptoms repeat CFM with frequent re-assessment of maternal status [14, 48].

Pre-term labor: Pre-term labor is a potential consequence of any traumatic event. Patients particularly at risk are those who sustained an occult abruption following direct uterine contusion, after fetal death, or following premature membrane rupture. With minor trauma, the risk has been described as less than 1\% [7]. With more severe trauma, the risk is greater approaching $28 \%$ in some series [52]. In the event of pre-term labor, alertness of the risk of occult abruption must be at a high level as further therapy is considered.

The standard medications used to treat pre-term labor all have a considerable unfavorable and unwanted side effect profile in the setting of acute trauma. Magnesium sulfate decreases respiratory efforts and, in high doses, may lead to hypotension, respiratory collapse, or cardiac arrhythmias. Terbutaline and other $\beta$-agonists cause cardiac stimulation leading to increased oxygen consumption, tachycardia, and hypotension. Such vital sign changes mimic those seen in occult hemorrhage, mandating scrutineous monitoring. Indomethacin affects platelet function, and is contraindicated in patient with head injury or occult bleeding. Calcium channel blockers may produce hypotension. Tocolytics should be reserved for those cases of true pre-term labor where the diagnosis is certain, the mother is well resuscitated, and continuous careful monitoring in an intensive care unit setting can be performed.

Uterine rupture: Uterine rupture, one of the most serious complications of blunt trauma in pregnancy, is a rather infrequent event in trauma in pregnancy. It occurs in less than $1 \%$ of blunt abdominal trauma and then most frequently in patients with a previous uterine scar [27]. Signs and symptoms are very variable from subtle uterine tenderness to frank shock. Owing to its frequent anterior location it is often associated with bladder rupture or bladder injury. Rupture of the unscarred uterus tends to occur posteriorly [2]. Uterine rupture carries a near $100 \%$ fetal mortality and $10 \%$ maternal mortality with most cases secondary to concomitant maternal injury [27].

Traumatic rupture of membranes: Rupture of membranes (ROM) secondary to trauma in pregnancy is seldom an isolated injury. A thorough search for concomitant injuries is mandatory and prolonged CFM strongly advocated. In the absence of maternal or fetal compromise management is usually not different from spontaneous ROM.

\section{Penetrating abdominal trauma}

Penetrating trauma in pregnancy is overall an infrequent event comprising of $3-10 \%$ of maternal trauma $[26,28,33]$. In inner city areas, however, new epidemiologic studies report much higher incidence rates 
making homicide due to penetrating trauma the number one cause of maternal mortality [53]. Visceral injuries due to penetrating trauma are significantly less common during pregnancy. Penetrating trauma in pregnancy due to gunshot wounds carries a $19 \%$ risk of visceral injury and a maternal death rate of $3.9 \%$ $[2,54]$. This is significantly different from the nonpregnant patient population where mortality rates of abdominal gunshot wounds have been reported between 10 and $12.5 \%$ and the risk of visceral injuries as high as $82 \%$ [2]. This protective effect against visceral injuries due to penetrating trauma in pregnancy is a consequence of the enlarging uterus occupying at the end of the third trimester as the largest intraperitoneal organ shielding half of the abdominal cavity [2]. However, fetal mortality can be as frequent as $70 \%$ which is a result of direct missile injury or the effects of prematurity $[2,55]$. Although not universally accepted, non-operative management has been advocated for penetrating trauma in pregnancy when the following prerequisites have been fulfilled:

1. The patient's vital signs are stable

2. There is no evidence of fetal distress, and
3. The anterior entry wound is certain to be below the uterine fundus.

However, the treating physician needs to be aware of the following:

1. The fetus is at risk with a prenatal mortality rate of up to $50 \%$ secondary to direct fetal injury or premature delivery.

2. Penetrating abdominal trauma involving the upper part of the peritoneal cavity is associated with an increase frequency of multiple visceral injuries. This is due to the compression of the small intestines into a smaller space.

It is important that such a trial of non-operative management for penetrating trauma in pregnancy is carried in high-volume trauma centers in close collaboration between experienced trauma surgeons and obstetrician. A diagnostic algorithm for the management of penetrating abdominal trauma in pregnancy is depicted in Fig. 2.

The following sections discuss new concepts and strategies of modern trauma management in pregnant patients:
Fig. 2 Penetrating abdominal trauma in pregnancy

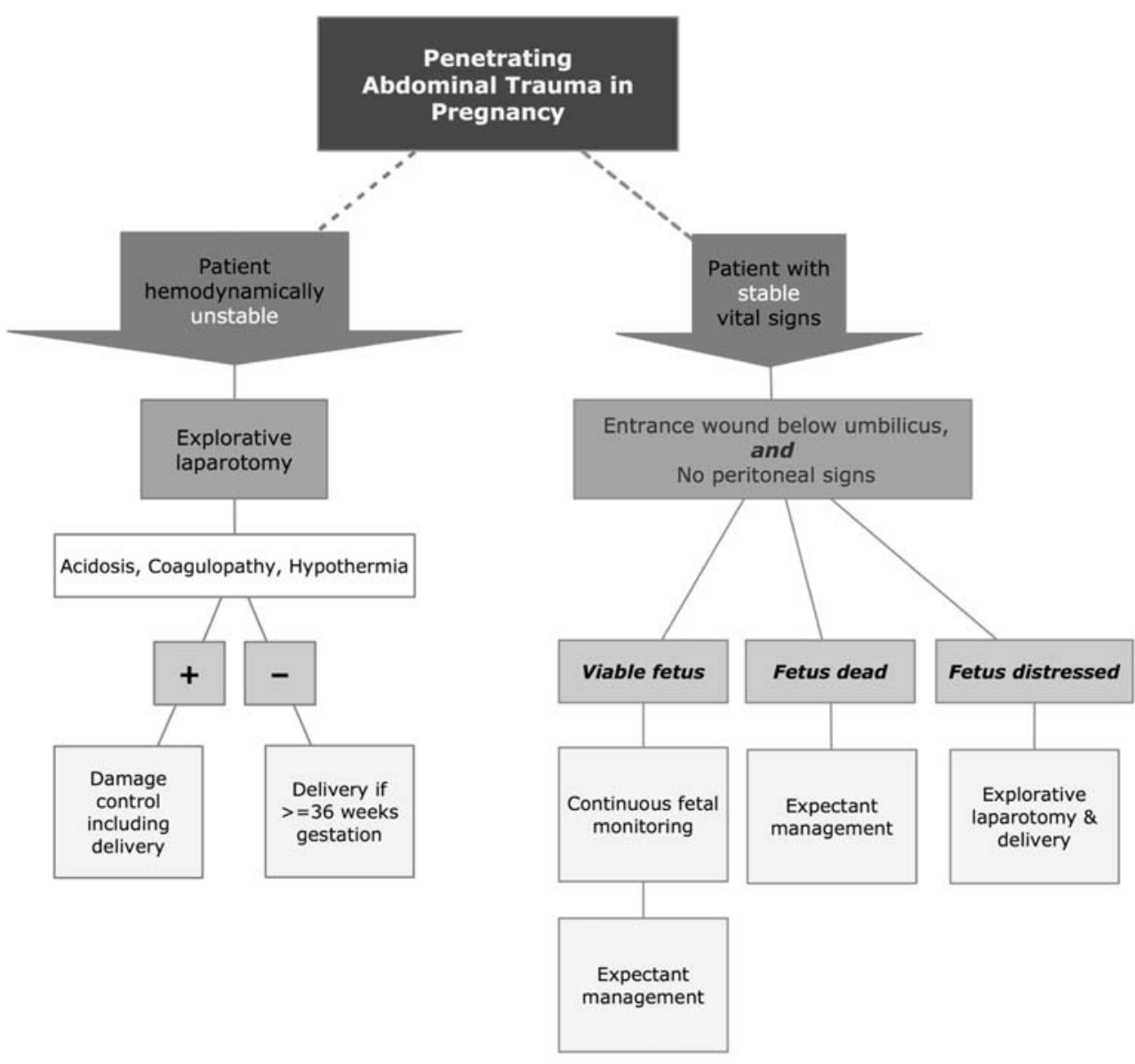




\section{Damage control surgery in pregnancy}

Damage control surgery is defined as rapid termination of an operation after control of life-threatening bleeding and intestinal spillage, followed by correction of physiologic abnormalities which precedes definitive management of initial injuries [56]. It is best defined as creating a stable anatomical environment to prevent the patient from progressing to an unsalvageable metabolic state when patients are more likely to die from metabolic failure than from failure to complete organ repair [57]. This modern strategy involves a staged approach to multiply injured patients. Damage control surgery is designed to avoid or correct the lethal triad of hypothermia, acidosis, and coagulopathy during or before definite injury management.

The concept of abbreviated laparotomy was first described by Stone in 1983 [58]. Any laparotomy was terminated with temporizing measures when coagulopathy was noted. These involved packing of the abdominal cavity in the majority of the cases to stop bleeding, and scheduled return to the operating room. The term "damage control" was popularized by Rotondo and Schwab, who in 1993 outlined a three-phase approach to patients with major abdominal injuries [59]. Phase one consisted of control of hemorrhage and contamination with rapid techniques of intra-abdominal packing and stapling-off intestinal ends, followed by temporary abdominal closure. Phase two in the ICU addressed restoration of a physiologic environment, in particular temperature, coagulation, and optimization of oxygen delivery. Phase three occurred, usually within $24-36 \mathrm{~h}$, with removal of abdominal packs, restoration of intestinal continuity, and definitive surgery with abdominal closure.

The concept of damage control was expanded further in 2001 by Johnson who added a forth phase at the beginning called "ground Zero" [60]. The principles of "ground zero" damage control include rapid transport to hospital and early decision making to facilitate hemorrhage control, prevention of hypothermia, and utilization of massive transfusion protocols. Since the early 1990s several series have consistently demonstrated superior survival rates of patients with blunt and penetrating trauma in whom principles of damage control surgery had been employed. Early considerations for damage control should be given in cases of

- Multi-system trauma with major abdominal injury

- Compound pelvic fracture with associated abdominal injury

- High-velocity gunshot or abdominal blast injury
- Penetrating abdominal injury with systolic blood pressure $\mathrm{SBP}<90 \mathrm{mmHg}$

Choosing the right patient for damage control is challenging. Awareness of potential triggers to initiate damage control is vital. Pre-emptive decision making to implement damage control should occur early rather than at a delayed point when the patient is in extremis. Accepted clinical and laboratory parameters for the application of principles of damage control surgery are the following:

- Hypotension: $\mathrm{SBP}<90 \mathrm{mmHg}$

- Hypothermia: $T<34^{\circ} \mathrm{C}$

- Coagulopathy: activated partial prothrombin time $(\mathrm{aPPT})>60 \mathrm{~s}$

- Acidosis: $\mathrm{pH}<7.2$ or arterial base deficit $(\mathrm{BE})<-8$

- Major intra-abdominal vascular injury

- Associated need for management of extra-abdominal life-threatening injury (e.g., concomitant thoracic injuries)

Reports on damage control surgery in pregnancy are rare and mostly limited to liver injuries. In one of the few and largest studies to date, Smith reviewed 35 cases of hepatic rupture in pregnancy [61]. Most of the cases were spontaneous occurrences complicated by HELLP syndrome. The maternal survival rate with packing and drainage was $82 \%$ compared to $25 \%$ in patients undergoing lobectomy. Delivery by cesarean section was carried out in nearly all cases. In case the patient required second packing due to persistent major hemorrhage from the liver at re-laparotomy, selective embolization during hepatic angiography was carried out at the conclusion of the second procedure [61]. Other more recent reviews are anectodal case reports or case series describing successful management of liver injuries with a planned staged approach (abbreviated laparotomy and scheduled return to the operating room) [62-64].

Pregnancy should not influence the decision to employ principles of damage control in severely injured woman. In fact, hypotension, coagulopathy, and acidosis, all which show or develop in pregnant woman at a later state, ask for a more proactive approach. Although not addressed in the recent literature, one of the main controversies of damage control surgery in the pregnant woman concerns the timing of delivery. Since most authorities agree on the fact that delivery of the fetus in maternal extremis should be part of resuscitation because of recruitment of the uteroplacental blood volume to the maternal circulation, delivery of a term or near-term fetus should be regarded as part of the damage control approach. The 
situation in the severely pre-term infant is more complex: assuming that damage control surgery is exclusively employed in catastrophic abdominal or thoracic injuries with ongoing bleeding, one would accept that the hemodynamic instability of the mother in these situations mandates the use of all possible resuscitative efforts including cesarean delivery of a premature fetus. However, that delivery of a preterm fetus is not always mandatory in the setting of damage control surgery shows a recent report by Aboutanos et al. [65]. A 20 -year-old gravida 1, woman sustained multiple gun shot wounds to the right chest and abdomen. The patient was 29 weeks pregnant. She underwent damage control surgery for her multiple intra-abdominal injuries with the abdomen kept open because of massive resuscitation requirements at the conclusion of the case. Fetal gestation could be safely prolonged until 32 weeks when the patient had a spontaneous vaginal delivery. During that time the abdomen was temporarily closed, initially with a vacuum pack technique, and then with abdominal skin only. Fascial retraction prevented primary closure after delivery and an elective abdominal hernia repair was scheduled. Fetal outcome was good [65]. This report demonstrates that in carefully selected cases continuation of pregnancy after initial successful application of principles of damage control surgery (control of bleeding and contamination, stabilization of vital signs, correction of acidosis, temporarily closed abdomen) is possible and can lead to favorable fetal outcome.

\section{Pelvic trauma in pregnancy}

Despite the occurrence of pelvic and acetabular fractures among women of childbearing age, literature specifically addressing patients who sustained pelvic or acetabular fractures in pregnancy is scarce. In a recent review, Leggon et al. [66] examined 101 cases of pelvic fractures (11 acetabular and 89 pelvic) in pregnant women. Overall, maternal mortality was $9 \%$ and fetal mortality was $35 \%$. Automobile-pedestrian collisions had a statistically higher maternal mortality rate than pregnant women involved in vehicular collisions which had a statistically significant trend towards a higher fetal mortality rate, as compared to falls. Most maternal deaths occurred from associated injuries, in particular from acute hemorrhage [66]. Injury severity influenced both maternal and fetal outcomes. Increasing injury severity (minor to moderate to severe) and associated injuries significantly increased both maternal and fetal death rates. Direct injury to placenta, uterus, or fetus accounted for fetal deaths in 52\%, while maternal hemorrhage (with or without maternal death) accounted for $36 \%$ of the non-surviving fetus. These observations are similar to predictors of fetal death after severe trauma in pregnancy in general. Predictors of fetal death in trauma in pregnancy include automobile-pedestrian collisions [67], motorcycle collisions [67], lack of restraints [67], maternal ejection during trauma [67, 68], and increased ISS [68, 69]. Mortality rates of women who sustained pelvic trauma in pregnancy were not affected by fracture classification (simple versus complex), fracture type (acetabular versus pelvic), the trimester of pregnancy, or the era studied by the review of literature [66, 69].

The close association between concomitant abdominal and pelvic injuries and fractures of the acetabulum or pelvis reflects the kinetic injury dissipated with the initial impact. Reported incidences of associated injuries in series of pregnant patients with pelvic fractures include the following rates: abdomen $42 \%$ (bladder $13 \%$, kidney $6 \%$, liver $6 \%$, spleen $5 \%$, urethra $5 \%$; closed-head injury, 37\%; thorax, $25 \%$; and most frequently, additional orthopedic injuries (fractures) in $48 \%$ of patients with pelvic fractures [69]. Overall, outcome of patients with pelvic fractures in pregnancy due to blunt trauma correlates with the severity of associated injuries and physiological derangement on admission rather than with characteristics of or the type of pelvic fracture. Occurrence of pelvic fractures in young patients requires transmission of significant amounts of kinetic energy and should therefore be regarded as an index injury mandating a thorough search for other occult visceral injuries.

Current guidelines recommend in the hemodynamically unstable, non-pregnant patient who sustained severe pelvic trauma and has no other identifiable source of bleeding (patients with negative DPL and/or FAST examination) control of pelvic or retroperitoneal hemorrhage via embolization of pelvic vessels during angiography, in particular of the hypogastric arteries [70]. Although successful pregnancies after prior occlusion of both hypogastric arteries have been described, there are no reports on the safety and efficacy of angioembolization for acute pelvic hemorrhage in pregnant patients.

Pelvic trauma in the third trimester of pregnancy should alert the trauma surgeon and obstetrician of direct injuries to the fetus. If the mother survives, fetal loss occurs in the majority of cases because of placental abruption, while direct injury to the fetus in the absence of uterine injury is a relatively infrequent event [71]. Direct injury of the fetus is usually associated with trauma occurring late in pregnancy, and most commonly involves fracture of the fetal skull [71]. This is 
especially likely during the third trimester when the fetal head is engaged low in the pelvis trapped between the anterior pelvic ring and the sacrum. The vast majority of in utero skull fractures have been related to severe maternal injury involving pelvic fractures [72]. Fetal skull fractures should be regarded as an index injury for severe maternal trauma. Vice versa, multiple pelvic fractures in pregnant women require a thorough sonoand radiographic examination of the uterus and fetus.

Pelvic and acetabular fracture surgery in pregnancy is performed infrequently [66, 72-74]. Acetabular fracture treatment was reported in 10 out of 12 cases, with skeletal traction and open reduction and internal fixation performed equally frequent [66]. Unstable fractures of the pelvic ring can be safely treated with open and percutaneous osteosynthetic techniques resulting in favorable pregnancy outcome [72-74]. A recent report describes the operative treatment of a vertical unstable fracture of the posterior pelvic ring using a low-exposure technique and imaging restricted to the posterior ring [74]

The recommended method of delivery for pelvic fractures during pregnancy depends on the presence of initial fetal and maternal distress, the degree of fetal maturity, the maternal injury severity, the displacement of the pelvic or acetabular fracture, and the eventual course of labor. Because these fractures tend to heal within 8 to 12 weeks, vaginal delivery should not be contraindicted after fractures that occurred earlier in pregnancy. Pubic rami fractures adjacent to the urethra or bladder, severe lateral compression fractures, and acute fractures of the pelvis with marked displacement may be relative indications for cesarean delivery if labor starts in a viable pregnancy. In the recent review by Leggon et al. [66], vaginal delivery was successful in $75 \%$ of pelvic fractures that occurred in the third trimester.

\section{Trauma scores and predictors of maternal and fetal outcomes}

Pre-hospital scores use primarily physiologic parameters and anatomic findings to help determine the appropriate level location for continued care. Such scoring systems allow caregivers in the field a somewhat objective list of criteria to use in directing transport of the injured victim. They assist in pre-hospital triage, ongoing clinical evaluation, and outcome evaluation. In 1980, the National Center for Health Services Research and the American Trauma Society determined that a scoring system should be simple to use, have predictive value, and show reliability between observers. For pregnant trauma victims, the most frequently employed and studied systems are the Revised Trauma Score (RTS) and the Injury Severity Score (ISS). Both systems have been shown to have limitations in pregnant trauma patients:

Maternofetal outcome has been found to correlate with the ISS [25]. Because it is usually assigned at discharge, however, this score has limited utility as a predictive tool. The ISS is a numeric quantification of traumatic injury in patients with one or more areas of the body involved and is specifically defined as the sum of the squares of the highest Abbreviated Injury Scale (AIS) grade in each of the three most severely injured areas' up to a maximum of 75 . With the normal maximum AIS score per organ system being 5 , the highest possible ISS for a person with traumatic injury to a single area of the body is 25 . The original study found that patients with an ISS of $<10$ rarely die and that those with one $>50$ rarely survive. Both, the AIS and the ISS score have been shown to be predictors of fetal outcome. Hoff et al. [24] found in a review on 73 pregnant trauma patients that fetal demise was found to be associated with increasing AIS and ISS, as well as with increasing fluid requirements, maternal acidosis and maternal hypoxia. Kissinger et al. [68] confirmed in a review on 93 pregnant trauma victims that ISS predicted which pregnancies were viable, while in general the maternal physiologic and laboratory parameters assessed failed to accurately predict pregnancy outcome. Since both AIS and ISS are anatomic scoring systems and require a definite diagnosis, their retrospective nature limits their value in predicting clinical outcome and influencing management decisions.

The RTS in comparison, assigned during the initial stages of all trauma resuscitation, is a composite measurement of Glasgow Coma Scale, blood pressure, and respiratory rate. Biester at al. [75] examined in a retrospective review of 30 pregnant trauma patients if a correlation exists between the RTS on admission and pregnancy outcome, and whether the RTS has predictive value for optimal duration of CTG monitoring to detect immediate adverse pregnancy outcome. In this small study, normal RTS on admission failed to detect in pregnant trauma patients intra-abdominal bleeds and placental abruption, and lacked predictive value for the need for prolonged CTG monitoring. Although the study is limited due to its retrospective nature and small sample seize, and the fact that many injuries were not severe enough to discern an abnormal RTS over a greater variety of injuries, the reported failure to detect adverse pregnancy outcome including fetal death supports the conclusion that a normal RTS is not an adequate measure predicting fetal outcome. 
Up to date statistically proven predictors of fetal death in pregnant trauma patients are elevated ISS, depressed maternal serum bicarbonate, low arterial $\mathrm{pH}$ and $\mathrm{pO} 2$, and severe maternal head injury [19, 25, 68]. These predictors, in combination with a high index of clinical suspicion, probably offer the best chance of detecting a potentially catastrophic impact on pregnancy outcome.

\section{The role of interpersonal violence}

Domestic violence against women has become increasingly recognized as a public health problem, and was declared a national epidemic by C. Everett Koop in 1992 [76]. Guth and Pachter [77] describe the scope of intentional interpersonal violence against women in a recent review as a hidden epidemic. In line with these observations, a large percentage of pregnant women experience significant trauma due to interpersonal violence during pregnancy [78]. Physical abuse of pregnant women is increasingly more recognized. This apparent increase may be a result of a truly increased incidence, or it may be a result of increased awareness, greater health care use, and better detection. Although most authors report an incidence of $10-15 \%$, a large series reported by Poole et al. [8] found an incidence of $31.5 \%$. Interpersonal violence against pregnant women appears to worsen as the pregnancy progresses through the second and third trimesters [79]. Regardless of the exact dynamics of pregnancy-related violence, most epidemiologic observational studies on that subject confirm that women who were abused while they were pregnant had a history of victimization in the past [78]. It also appears that the psychosocial stressors of pregnancy are causal in many of these cases [80]. Risk factors for pregnancy-related violence include low socioeconomic status, low levels of social support, first time parenting, carrying an unexpected or unwanted pregnancy, race/ ethnicity, older age, teenage pregnancy, and substance abuse (Table 2) [78]. Consequences of pregnancyrelated violence include delayed prenatal care, low birth weight, small for gestational age fetus, anemia, and increased risk of drug and alcohol use and poor health care (Table 3) [78, 81, 82]. Spouses and boyfriends are the perpetrators in $70-85 \%$ of the cases [81]. Interpersonal violence is a clear marker for adverse maternal and fetal outcomes.

Physical abuse in pregnancy is more likely to manifest with proximal and midline injuries than the distal injuries of accidental trauma. Injuries to the neck and face are most common [83]. Trunk bruises and injuries
Table 2 Correlates of pregnancy-related violence

Low socioeconomic status

Low levels of social support

First time parenting

Unplanned or unwanted pregnancy

Race/ethnicity

Older age

Youth

Alcohol/substance abuse

Table 3 Consequences of pregnancy-related violence

Delayed prenatal care

Low birth weight

Premature labor

Increased risk of operative delivery

Fetal trauma

Health issues for mother

to the upper arm and lateral thighs are common [81, 83]. Cigarette burns and bites should not be overlooked since these injuries are pathognomonic for domestic violence.

Maternal and fetal morbidity and mortality due to physical abuse in pregnancy varies according to the nature and severity of the inflicted injury. Associations between maternal morbidity and physical violence during pregnancy includes a 3.3-fold increased incidence in placental abruption and a 3.5-fold increased risk of pre-term labor [81, 82]. Women experiencing violence are more frequently hospitalized antenatally and were twice as likely to deliver by cesarean section [82]. Acutely life-threatening injuries reported in association with interpersonal violence in pregnancy include uterine rupture and splenic injury due to abdominal kicks and blows [81, 82, 84]. Fetal morbidity and mortality is largely determined by the degree of asphyxia, fetal distress, and prematurity following pre-term delivery.

The most effective strategies for identifying domestic violence tend to incorporate multiple in-person interviews by highly trained individuals asking multiple direct questions $[13,85]$. Using a simple five-question screening tool (Fig. 3), McFarlane et al. [10] found a $17 \%$ prevalence of physical or sexual abuse during pregnancy, a more than twofold increase from previous studies. Sixty percent of abused pregnant women reported two or more occurrences [13]. Recognizing the magnitude of the problem, in 1994 the Joint Hospital Accreditation Manual under the section 'Patient Assessment' advised that criteria are to be developed by each institution to help identify probable victims of abuse [86]. The ACOG and RCOG have published guidelines for the screening of abuse and domestic 
Fig. 3 Abuse Assessment Screen. Women who answered yes to questions 2,3 , or 4 were considered abused
Abuse Assessment Screen (Circle YES or NO for each questions)

1. Have you ever been emotionally or physically abused by your partner or somebody important to you $? \ldots \ldots \ldots \ldots \ldots \ldots \ldots \ldots \ldots \ldots$ YES NO

2. Within the last year, have you been hit, slapped, kicked, or otherwise physically

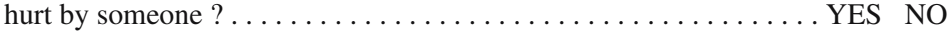
If YES, by whom (circle all that apply) Husband ex-husband Boyfriend Stranger Other Total No. of times

3. Since you have been pregnant, have you been hit, slapped, kicked, or otherwise physically hurt by someone $? \ldots \ldots \ldots \ldots \ldots \ldots \ldots \ldots$ YES NO If YES, by whom (circle all that apply)

Husband ex-husband Boyfriend Stranger Other Total No. of times

Mark the area of injury on a body map.

Score each incident according to the following scale: $1=$ Threats of abuse, including use of a weapon $2=$ Slapping, pushing; no injuries and/or lasting pain $3=$ Punching, kicking, bruises, cuts, and/or continuing pain $4=$ Beaten up, severe contusions, burns, broken bones $5=$ Head, internal, and/or permanent injury $6=$ Use of weapon, wound from weapon

(If any of the descriptions for the higher number apply, use the higher number)

4. Within the last year, has anyone forced you to have sexual activities ?.......

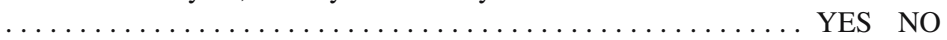

If YES, by whom (circle all that apply)

Husband Ex-husband Boyfriend Stranger Other Total No. of times

5. Are you afraid of your partner or anyone you listed above ?..... YES NO violence, and routine screening is now integral part of antenatal care in many centers [87, 88]. This includes screening for domestic violence among pregnant women who present with non-trauma-related symptoms to hospital emergency departments [89]. Such visits should be regarded as an opportunity for all health care providers to detect domestic violence since it might be the only environment women feel safe to reveal their abuse $[1,89,90]$. If domestic violence is suspected, other ancillary services, such as social services and law enforcement, should be involved immediately since undiagnosed and untreated domestic violence poses a series risk to both the mother and the fetus. Inpatient care may allow better evaluation and assessment of the home environment and allows planning for possible necessary escape plans.

\section{References}

1. Connolly AM, Katz VL, Bash KL et al (1997) Trauma and pregnancy. Am J Perinatol 14:331-335

2. Shah AJ, Kilcline BA (2003) Trauma in pregnancy. Emerg Med Clin North Am 21:615-629

3. Fildes J, Reed L, Jones N et al (1992) Trauma: the leading cause of maternal death. J Trauma 32:643-645
4. Rothenberger D, Quattlebaum FW, Perry JF et al (1978) Blunt maternal trauma: A review of 103 cases. J Trauma 18:173-179

5. Goldman SM, Wagner LK (1996) Radiologic management of abdominal trauma in pregnancy. Am J Radiol 166:763-767

6. Hill DA, Lense JJ (1996) Abdominal trauma in the pregnant patient. Am Fam Physician 53:1269-1274

7. Dahmus M, Sibai B (1993) Blunt abdominal trauma: are there any predictive factors for abruption placentae or maternal-fetal distress? Am J Obstet Gynecol 169:1054-1059

8. Poole GV, Martin JN, Perry KG et al (1996) Trauma in pregnancy: The role of interpersonal violence. Am J Obstet Gynecol 174:1502-1510

9. Newberger EH, Barkan SE, Lieberman ES et al (1992) Commentary: abuse of pregnant women and adverse birth outcome. Current knowledge and implications for practice. JAMA 267:2370-2372

10. McFarlene J, Parker B, Soeken K et al (1992) Assessing for abuse during pregnancy. JAMA 267:3176-3178

11. McLeer SV, Anwar R (1989) A study of battered women presenting in an emergency department. Am J Public Health 79:65-66

12. Joint Commission on Accreditation of Healthcare Organizations: Accreditation Manual for Hospitals, 1993, p 6

13. Gazmararian J, Lazorick S, Spitz A et al (1996) Prevalence of violence against pregnant women. JAMA 275:915-920

14. Barraco RD, Chiu WC, Clancy TV et al Practice management guidelines for the diagnosis and management of injury in the pregnant patient: the EAST Practice Management Guidelines Work Group. The Eastern Association for the Surgery of Trauma 2005; http://www.east.org/ 
15. Goodwin T, Breen M (1990) Pregnancy outcome and fetomaternal hemorrhage after non-catastrophic trauma. Am J Obstet Gynecol 162:665-671

16. Schneider R (2000) Muscle relaxants. In: Walls R (ed) Emergency airway management. Lippincott Williams Wilkin, Philadelphia, pp 121-128

17. Rees GA, Willis BA (1988) Resuscitation in late pregnancy. Anaesthesia 43:347-349

18. Kasten GW, Martin ST (1986) Resuscitation from bupivacaine-induced cardiovascular toxicity during partial inferior vena cava occlusion. Anesth Analg 65:341-344

19. Lavery JP, Staten-McCormick M (1995) Management of moderate to severe trauma in pregnancy. Obstet Gynecol Clin North Am 22:69-90

20. Archer GW, Marx GF (1974) Arterial oxygen tension during apnoea in parturient women. Br J Anaesth 46:358-360

21. Pearlman MD, Tintinalli JE, Lorenz RP (1990) Blunt trauma during pregnancy. N Engl J Med 323:1609-1613

22. Segal S, Shemesh IY, Blumenthal R et al (2003) Treatment of obstetric hemorrhage with recombinant activated factor VII (rFVIIa). Arch Gynecol Obstet 268:266-267

23. Dutton RP, Hess JR, Scalea TM (2003) Recombinant factor VIIa for control of hemorrhage: early experience in critically ill trauma patients. J Clin Anesth 15:184-188

24. Hoff WS, D'Amelio LF, Tinkoff GH et al (1991) Maternal predictors of fetal demise in trauma during pregnancy. Surg Gynecol Obstet 172:175-180

25. Scorpio R, Esposito T, Smith G et al (1992) Blunt trauma during pregnancy: factors affecting fetal outcome. J Trauma 32:213-216

26. Shah KH, Simons RK, Holbrook T et al (1998) Trauma in pregnancy: maternal and fetal outcomes. J Trauma 45:83-86

27. Pearlman MD, Tintinalli J (1991) Evaluation and treatment of the gravida and fetus following trauma during pregnancy. Obstet Gynecol Clin North Am 18:371-381

28. Esposito TJ, Gens DR, Smith LG et al (1991) Trauma during pregnancy: a review of 79 cases. Arch Surg 126:1073-1078

29. Gentilello KM, Samuels PN, Henningfield JE et al (2005) Alcohol screening and intervention in trauma centers: confidentiality concerns and legal considerations. J Trauma 59:1250-1254

30. American College of Obstetricians and Gynecologists (1998) Obstetric Aspects of Trauma Management, vol 251. ACOG Educational Bulletin. Int J Gynaecol Obstet 1999; 64:87-94

31. Katz VL, Dotters DJ, Droegemueller W (1986) Perimortem cesarean delivery. Obstet Gynecol 68:571-576

32. Lanoix R, Akkapeddi V, Goldfeder B (1995) Perimortem cesarean section: case reports and recommendations. Acad Emerg Med 2:1063-1067

33. Morris JA, Rosenbower TJ, Jurkovich GJ et al (1996) Infant survival after cesarean section for trauma. Ann Surg 223:481491

34. Lee RV, Rodgers BD, White LM et al (1986) Cardiopulmonary resuscitation of pregnant women. Am J Med 81:311-318

35. Crosby WM, Costiloe JP (1971) Safety of lap-restraint for pregnant victims of automobile collisions. N Engl J Med 284:632-636

36. Johnson HC, Pring DW (2000) Car seatbelts in pregnancy: the practice and knowledge of pregnant women remain causes for concern. BJOG 107:644-647

37. American College of Obstetricians and Gynecologists (1991) Automobile passenger restraints for children and pregnant women, vol 151. ACOG Technical Bulletin. Int J Gynaecol Obstet 1992; 37:305-308

38. Royal College of Obstetricians and Gynaecologists (2004) Why mothers die 2000-2002. The sixth report of the confiden- tial enquiries into maternal deaths in the United Kingdom. RCOG Press, London

39. Pearlman MD, Tintinalli JE, Lorenz RP (1990) A prospective controlled study of outcome after trauma in pregnancy. Am J Obstet Gynecol 162:1502-1510

40. Moise KJ Jr, Belfort MA (1997) Damage control for the obstetric patient. Surg Clin North Am 77:835-852

41. Smith LG, Moise KJ, Dildy GA et al (1991) Spontaneous rupture of liver during pregnancy: Current Therapy. Obstet Gynecol 77:171

42. Harris BM, Kuczkowski KM (2005) Diagnostic dilemma: hepatic rupture due to HELLP syndrome vs. trauma. Arch Gynecol Obstet 272:176-178

43. Pearlman MD, Viano D (1996) Automobile crash simulation with the first pregnant crash test dummy. Am J Obstet Gynecol 175:977-981

44. Ma O, Mateer J, DeBehnke D (1996) Use of ultrasonography for the evaluation of pregnant trauma patients. J Trauma 40:665-668

45. Goodwin H, Holmes J, Wisner D (2001) Abdominal ultrasound examination in pregnant blunt trauma patients. J Trauma 50:689-694

46. Higgins SD, Garite TJ (1984) Late abruptio placentae in trauma patients: implications for monitoring. Obstet Gynecol 63:10S

47. Esposito TJ, Gens DR, Smith LG et al (1989) Evaluation of blunt abdominal trauma during pregnancy. J Trauma 29:1628-1632

48. Towery R, English P, Wisner D (1993) Evaluation of pregnant women after blunt injury. J Trauma 35:731-736

49. Pearlman MD, Tintinalli JE, Lorenz RP (1990) A prospective controlled study of outcome after trauma during pregnancy. Am J Obstet Gynecol 162:1502-1507

50. Nolan TE, Smith RP, Devoe LD (1993) A rapid test for abruptio placentae: evaluation of a D-dimer latex agglutination slide test. Am J Obstet Gynecol 169:265-269

51. Emery CL, Morway LF, Chung-Park M et al (1995) The Kleihauer-Betke test: clinical utility indication, and correlation in patients with placental abruption and cocaine use. Arch Pathol Lab Med 119:1032-1037

52. Williams JK, McClain L, Rosemurgy AS et al (1990) Evaluation of blunt abdominal trauma in the third trimester of pregnancy. Obstet Gynecol 75:33-37

53. Chang J, Berg CJ, Saltzman LE et al (2005) Homicide: a leading cause of injury deaths among pregnant and postpartum women in the United States, 1991-1999. Am J Public Health 95:471-477

54. Awwad JT, Azar GB, Seoud MA et al (1994) High-velocity penetrating wounds of the gravid uterus: review of 16 years of civil war. Obstet Gynecol 83:259-264

55. Franger AL, Buschsbaum HJ, Peaceman AM et al (1989) Abdominal gunshot wounds in pregnancy. Am J Obstet Gynecol 160:1124-1128

56. Schreiber MA (2004) Damage control surgery. Crit Care Clin 20:101-118

57. Sugrue M, D'Amours SK, Joshipura M (2004) Damage control surgery and the abdomen. Injury 35:642-648

58. Stone HH, Strom PR, Mullins RJ (1983) Management of the major coagulopathy with onset during laparatomy. Ann Surg 197:532-535

59. Rotondo M, Schwab C, McGonigal M et al (1993) Damage control: an approach for improved survival in exsanguinating penetrating abdominal injury. J Trauma 35:375-382

60. Johnson J, Gracias V, Gupta R et al (2002) Hepatic angiography in patients undergoing damage control laparatomy. $\mathrm{J}$ Trauma 52:1102-1106 
61. Smith LG, Moise KJ, Dildy GA et al (1991) Spontaneous rupture of liver during pregnancy: Current therapy. Obstet Gynecol 77:171

62. Muench MV, Baschat AA, Dorio PJ et al (2004) Successful pregnancy outcome after splenic artery embolization for blunt maternal trauma. J Trauma 56:1146-1148

63. Merchant SH, Mathew P, Vanderjagt TJ et al (2004) Recombinant factor VIIa in management of spontaneous subcapsular liver hematoma associated with pregnancy. Obstet Gynecol 103:1055-1058

64. Reck T, Bussenius-Kammerer M, Ott R et al (2001) Surgical treatment of HELLP syndrome-associated liver rupture-an update. Eur J Obstet Gynecol Reprod Biol 99:57-65

65. Aboutanos SZ, Aboutanos MB, Malhotra AK et al (2005) Management of a pregnant patient with an open abdomen. J Trauma 59:1052-1055

66. Leggon RE, Wood GC, Indeck MC (2002) Pelvic fractures in pregnancy: factors influencing maternal and fetal outcomes. $\mathrm{J}$ Trauma 53:796-804

67. Curet MJ, Schermer CR, Cemarest GB et al (2000) Predictors in outcome in trauma during pregnancy: identification of patients who can be monitored for less than 6 hours. J Trauma 49:18-24

68. Kissinger DP, Rozycki GS, Morris JA et al (1991) Trauma in pregnancy: predicting pregnancy outcome. Arch Surg 126:1079-1086

69. Parreira JG, Coimbra R, Rasslan S et al (2000) The role of associated injuries on outcome of blunt trauma patients sustaining pelvic fractures. Injury 31:677-682

70. DiGiacomo JC, Bonadies JA, Cole FJ et al: Practice management guidelines for hemorrhage in pelvic fracture: the EAST Practice Management Guidelines Work Group. The Eastern Association for the Surgery of Trauma 2005; http:// www.east.org/

71. Stafford PA, Biddinger PW, Zumwalt RE (1988) Lethal intrauterine fetal trauma. Am J Obstet Gynecol 159:485-489

72. Pape HC, Pohlemann T, Gansslen A et al (2000) Pelvic fractures in pregnant multiple trauma patients. J Orthop Trauma $14: 238-244$

73. Dunlop DJ, McCahill JP, Blakemore ME (1997) Internal fixation of an acetabular fracture during pregnancy. Injury 28:481-482

74. Loegters T, Briem D, Gaztka C et al (2005) Treatment of unstable fractures of the pelvic ring in pregnancy. Arch Orthop Trauma Surg 125:204-208
75. Biester EM, Tomich PG, Esposito TJ et al (1997) Trauma in pregnancy: normal revised trauma score in relation to other markers of maternofetal status - a preliminary study. Am J Obstet Gynecol 176:1206-1210

76. Sisley A, Jacobs LM, Poole G et al (1999) Violence in America: a public health crisis-domestic violence. J Trauma 46:1105-1113

77. Guth AA, Pachter HL (2000) Domestic violence and the trauma surgeon. Am J Surg 179:134-140

78. Jasinski JL (2004) Pregnancy and domestic violence: a review of the literature. Trauma Violence Abuse 5:47-64

79. Newberger EH, Barkan SE, Lieberman SE et al (1992) Commentary: abuse of pregnant women and adverse birth outcome. JAMA 267:2370

80. Mighty H (1994) Trauma in pregnancy. Crit Care Clin 10:623-634

81. Parker B, McFarlane J, Soeken K (1994) Abuse during pregnancy: effects on maternal complications and birth weights in adults and teenage women. Obstet Gynecol 84:323-328

82. Rachana C, Suraiya K, Hisham AS et al (2002) Prevalence and complications of physical violence during pregnancy. Eur J Obstet Gynecol Reprod Biol 103:26-29

83. Campbell JC, Sheridan D (1989) Emergency nursing interventions with battered women. J Emerg Nurs 15:12

84. McKenna D, Adair S, Price J (2003) Domestic violence in late pregnancy and splenic injury: delayed presentation. Acta Obstet Gynecol Scand 82:95-96

85. Price S, Baird K, Salmon D (2005) Asking the question: antenatal domestic violence. Pract Midwife 8:21-25

86. Joint Commission on Accreditation of Healthcare Organizations (1993) Accreditation Manual for Hospitals, p 6

87. American College of Obstetricians and Gynecologists (ACOG) (1995) Domestic violence, vol 209. ACOG Technical Bulletin. Int J Gynaecol obstet 1995; 209:1-9

88. Bewley S, Friend J, Mezey G (eds) (1997) Violence against women. RCOG Press, London

89. Dienemann J, Trautman D, Shahan JB et al (1999) Developing a domestic violence program in an inner-city academic health center emergency department: the first 3 years. J Emerg Nurs 25:110-115

90. Koss MP, Koss PG, Woodruff J (1991) Deleterious effects of criminal victimization on women's health and medical utilization. Arch Int Med 151:342-347 